\title{
A COMPENSATED IMPEDANCE BASED GROUND FAULT LOCATOR FOR AN UNEARTHED POWER SYSTEM \\ Hayder K. Jahanger ${ }^{1,2}$, Abdurrahman Raqib ${ }^{1}$, David W.P. Thomas ${ }^{1}$, Mark Sumner ${ }^{1}$ \\ ${ }^{1}$ PEMC Group, University of Nottingham, Nottingham, UK \\ ${ }^{2}$ Department of Electrical Engineering, University of Technology, Baghdad, Iraq \\ *ezahj4@nottingham.ac.uk
}

Keywords: COMPENSATION, FAULT LOCATION, IMPEDANCE, UNEARTHED SYSTEM

\begin{abstract}
Continuous operation of a power system during a ground fault can be achieved by employing either a High Resistance Grounded (HRG) power source or a floating power source. However, if a second ground fault occurs in the system it can cause significant problems and therefore the presence of the First Ground Fault (FGF) must be detected, located, and repaired quickly. In this study, an active impedance based fault location method is proposed to locate the FGF in a HRG power system. The study presented shows that the magnitude of the fault resistance has only a small influence on the proposed technique. However, for unbalanced faults, the impedance of the healthy phases can distort the impedance measured and impair the accuracy of the fault location method. Therefore, a new compensated method is proposed to compensate for this distortion. The results show that a high accuracy can be achieved with the compensation, with a location error lower than $5 \%$.
\end{abstract}

\section{Introduction}

The use of High-Resistance Ground (HRG) or ungrounded supplies in Marine power systems has shown many benefits compared to the use of low-resistance or solidly grounded systems. The most important benefit is the ability for continuous power flow even in the presence of a single (first) fault to ground [1]. This is from the perspective of power quality and management. Moreover, this grounding technique also helps in minimising possible arc events (that may lead to damage of electrical apparatus) $[1,2,3]$ reducing outage time, production losses and equipment damage [4].

The major issue with this type of grounding is that if the first ground fault has is not been cleared, a second ground fault leads to a phase-to-phase fault condition with a high fault current or an arcing hazard. Consequently, the time to detect and locate the first ground fault (FGF) is highly important. However, locating the FGF is challenging for traditional fault location techniques that use the fault current and voltage to estimate the impedance to the fault location [4].

Recent research on fault location in HRG or ungrounded power systems has been published in [4-13]. The authors in [5] used the voltage measurement at source end to locate the fault. The Wavelet Transform (WT) with multi-resolution analysis (MRA) is utilised to extract specific patterns for each fault location from system fundamental and the background noise. However, the technique requires a zonal system configuration as the fault location strategy is based on a zonal pattern. Experimental validation of the technique is presented in [6], where the inherent high-frequency signals associated with the switching events of converters is used to locate the fault. Another drawback of this method is the requirement of measurements in a relatively high frequency range (over 100
$\mathrm{kHz}$ ). The influence of the converter's coupling capacitance on the accuracy of the background noise pattern is assessed in $[7,8]$. The study showed that the coupling capacitance has a potential influence on the scheme that requires the used pattern to be changed for different coupling capacitance values. The study also showed that the WT requires a high-frequency decomposition band. A different technique is suggested in [9]. In this research, the authors used the midpoint voltage measurement on the DC side of an AC-DC-AC converter to identify and locate a line-to-ground fault. The algorithm is based on comparing the measured DC Voltage (Vng) to three possible voltages in the system (line side, $\mathrm{dc}$ side and drive side). If the measured voltage is similar to one of these voltages, the fault is at that location in the system [9]. However, this technique is not tested on a distribution system with different configurations. The same author presented a self-diagnosis algorithm for multiple converters in a HRG system. The disadvantage of proposed technique is the complex hardware and high-level hardware/software required [10]. Single-ended and two ended approaches are proposed to locate faults on multi-phase systems which include laterals in [11]. The faulted section is firstly detected, and then the fault position is located through detailed circuit and topology analysis of the faulty section. However, the technique requires measurements from several different terminals.

Active fault location methods have been proposed by other researchers in order to improve the signal to noise ratio of measurements made when trying to identify and locate a FGF. Active impedance estimation is used to locate the fault in the HRG integrated power system presented in [12]. Although the technique showed reliable outcomes, the study did not consider a proper resolution for high load powers. The authors suggested switching off the high load during the fault location process. Moreover, this study suggested a method based on 
impedance measurements but not influenced by the system load [13]. However, the method requires multiple measurements throughout the system to increase the accuracy, and a communication channel is essential between the measurement units and the central processing unit. The authors used an injected (5A to $10 \mathrm{~A}$ ) current pulse operating at the tenth harmonic $(600 \mathrm{~Hz})$ to locate the FGF in a HRG system in [4]. The fault is located by monitoring and sensing the injected pulse. However, the accuracy of the scheme requires a high number of sensing devices throughout the system which increases the installation cost. Another study suggested the utilisation of a two-ended impedance based method [14]. Though this method offers a good accuracy, it required two sets of synchronised voltage and current measurements for each protected line.

This paper presents a direct and precise method for first ground fault location in HRG integrated power systems which uses measurement of the ground voltage and current i.e. it is a single ended impedance based method. The work also suggests a new technique to overcome the influences of high load levels and the non-faulted phases on the accuracy of the estimated fault location. The single-ended estimation method operates at nonfundamental frequencies and are shown to locate the first ground fault with errors of less than $6 \%$.

\section{The Proposed FGF Location Technique}

The non-compensated and compensated active first ground fault location techniques applied to a HRG power system will be introduced in this section.

\subsection{The non-compensated approach}

A simplified integrated power system such as the type found in a naval power system with a HRG power source and floating load is shown in Fig. 1 and is used to describe the algorithm. $\mathrm{Z}_{\text {ground }}$ is approximately $100 \mathrm{k} \Omega$ for this study. The source supplies a floating three-phase load through a three-phase distribution line and a has a source impedance. If a FGF is applied on phase $\mathrm{c}$ at the midline as shown in Fig. 1, it can be detected by continuously monitoring the voltage measurement at the HRG source terminals. When the FGF occurs, one of the measured line to ground voltages reduces significantly (but the current is limited to small values by $\mathrm{Z}_{\text {earth }}$ ), and this change can be used to detect the presence of the FGF. When the fault is detected, a small resistance $R_{\text {switch }}$ is switched in parallel to the HRG after $10 \mathrm{~ms}$ for a period of $30 \mu \mathrm{s}$ in order to create a current and voltage transient: this provides information for fault location over a wide frequency range. The equivalent circuit during this period, at non-fundamental frequencies is shown in Fig. 2 [12].

where $Z_{s-a}, Z_{s-b}$ and $Z_{s-c}$ are the source impedances per phase, $Z_{x-a}, \quad Z_{x-b}, \quad Z_{x-c}$ are the impedance to the fault location for each phase, while $Z_{l-x a}, Z_{l-x b}, Z_{l-x c}$ are the remaining line impedances. $Z_{\text {load-a }}, Z_{\text {load }-b}$ and $Z_{\text {load }-c}$ are the load impedance for each phase. The transient voltage $\mathrm{V}_{\text {switch }}$ in Fig. 2 is the voltage generated due to the switching of the small parallel resistance in the ground path. Both $I_{\text {switch }}$ and $V_{\text {switch }}$ are measured during the $30 \mu$ s transient.

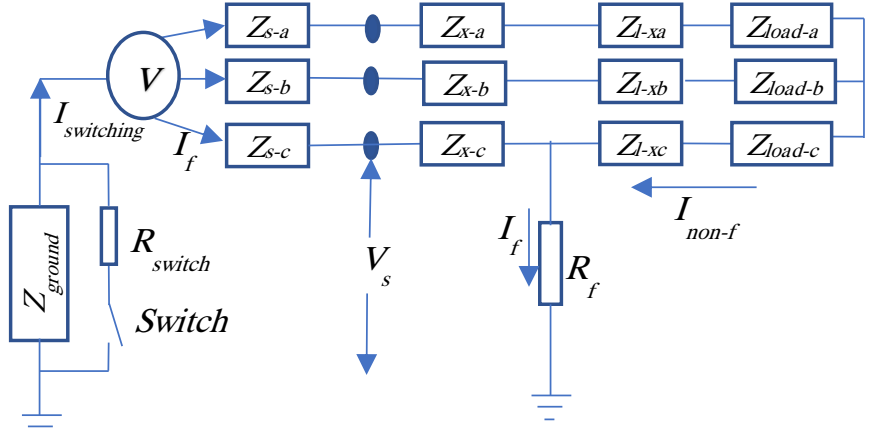

Fig. 1 A first ground fault in a HRG three-phase system

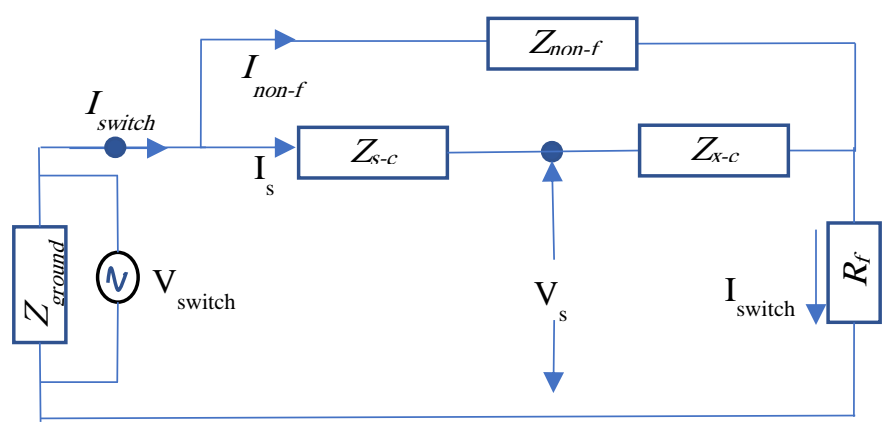

Fig. 2 The system at non-fundamental frequencies during the switching of the parallel resistance

The switched transient current flows through both the faulted $\left(Z_{s-c}\right.$ and $\left.Z_{x-c}\right)$ and healthy cable $\left(Z_{n o n-f}\right)$ to the fault point. $Z_{\text {non-f }}$ is calculated as following:

$Z_{\text {non-f }}=Z_{T}+Z_{\text {load }-c}+Z_{l-x c}$

where $Z_{T}$ is the equivalent impedance of the healthy phases and described as

$Z_{T}=\left(Z_{s-a}+Z_{x-a}+Z_{l-x a}\right) \|\left(Z_{s-b}+Z_{x-b}+Z_{l-x b}\right)$

The majority of the switched transient current will flow through the faulted phase if $Z_{n o n-f}$ is significantly larger than $\left(Z_{s-c}\right.$ and $\left.Z_{x-c}\right)$. Hence, the part that flows through $Z_{n o n-f}$ is usually negligible. The distance between the fault location and the source end is calculated as:

$X_{x-c}=\frac{\operatorname{imag}\left(\frac{F F T\left(V_{\text {Switch }}\right)}{F F T\left(I_{\text {switch }}\right)}-Z_{s-c}\right)}{X_{\text {Per-metre }}}$

$Z_{s-c}$ can be estimated using measurements made immediately before the fault occurs at the source terminals. Consequently, removing the FGF as quickly as possible will prevent the possibility of a high fault current that could be created due to a second ground fault.

\subsection{The compensated approach}

A significant part of the transient current will pass through $Z_{\text {non-f }}$ whenever $Z_{\text {non-f }}$ is comparable to the impedance of the faulted branch - this is particularly true when the FGF 


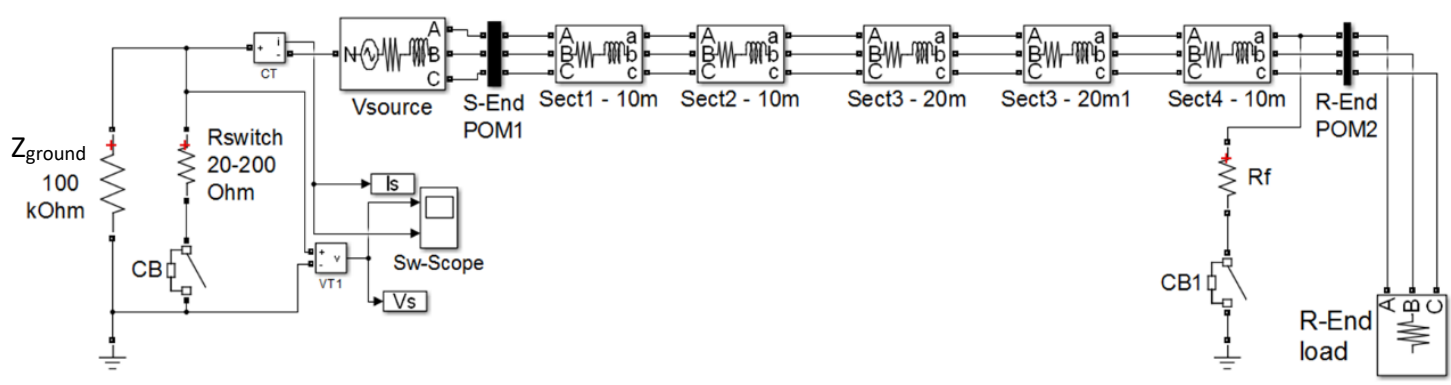

Fig. 3 Simulated three phase system with a HRG source

impedance is high. As a result of this condition, (3) will lead to a high estimation error. To solve this issue, a new compensation technique is proposed to compensate for the error present due to the current passing through $Z_{\text {non-f }}$. A derivation of the method is presented below using the equivalent circuit of Fig. 2.

$V_{\text {switch }}=Z_{s-c} * I_{s}+Z_{x-c} * I_{s}+R_{f} * I_{f}$

But, $I_{s}=I_{\text {switch }}-I_{\text {non-f }}$, then

$V_{\text {switch }}=Z_{s-c} *\left(I_{\text {switch }}-I_{\text {non }-f}\right)+Z_{x-c} *\left(I_{\text {switch }}-\right.$

$\left.I_{\text {non-f }}\right)+R_{f} * I_{\text {switch }}$

Dividing (5) by $I_{\text {switch }}$ yields,

$\frac{V_{\text {switch }}}{I_{\text {switch }}}=Z_{s-c} *\left(1-\frac{I_{\text {non-f }}}{I_{\text {switch }}}\right)+Z_{x} *\left(1-\frac{I_{\text {non }-f}}{I_{\text {switch }}}\right)+R_{f}$

Rearrange (6) for $X_{x-c}$, gives (7),

$X_{x-c}=\operatorname{Imag}\left[\frac{\frac{F F T\left(V_{\text {switch }}\right)}{F F T\left(I_{\text {switch }}\right)}-z_{s-c}\left(1-\frac{I_{\text {non-f }}}{I_{\text {Switch }}}\right)}{\left(1-\frac{I_{\text {non-f }}}{I_{\text {Switch }}}\right)}\right]$

where, $\quad \frac{I_{\text {non }-f}}{I_{\text {switch }}}=\frac{Z_{s-c}+Z_{x-c}}{Z_{s-c}+Z_{x-c}+Z_{n o n-f}}$

Finally, an iterative approach is required to apply (7) and (8) as follows:

1) In the beginning, pre-fault measurement is used to estimate the source impedance and the load.

2) $Z_{x-c}$ is assumed to equal Zero, and an initial value for $\left(\frac{I_{\text {non-f }}}{I_{\text {switch }}}\right)$ using (8) is calculated.

3) Then, (7) is used to estimate a new value for $X_{x-c}$ and a new fault location.

4) $X_{x-c}$ calculated in step (3) is used to update $\left(\frac{I_{\text {non-f }}}{I_{\text {switch }}}\right)$ using (8).

5) Again, $X_{x-c}$ is updated using (7).

6) This iteration is continued until the distance between two successive iterations converges to a pre-set tolerance such as $0.5 \mathrm{~m}$.

\section{Verification Through Simulation}

A three-phase integrated power system has been simulated in Matlab/Simulink as shown in Figure 3. The system consists of the primary source grounded with high resistance $\left(Z_{\text {ground }}\right)$, a three-phase transmission line, and a floating three-phase load. The transmission line is divided into five equal sections of 10 metres. Further details are presented in Table 1. The measured line to ground voltage is used to detect the presence of the FGF, and after $10 \mathrm{~ms}, \mathrm{R}_{\text {switch }}$ is switched into the ground circuit for $30 \mu \mathrm{s}$. An example of the measured voltage and current are shown in Fig. 4 with two different $\mathbf{R}_{\text {switch }}$ values. The lower impedance creates a higher magnitude transient injection. Fig. 5 shows another measurement but with two different fault resistances. This Figure shows that the fault resistance has a very small influence on the location technique for these low values of $\mathbf{R}_{\text {switch. }}$
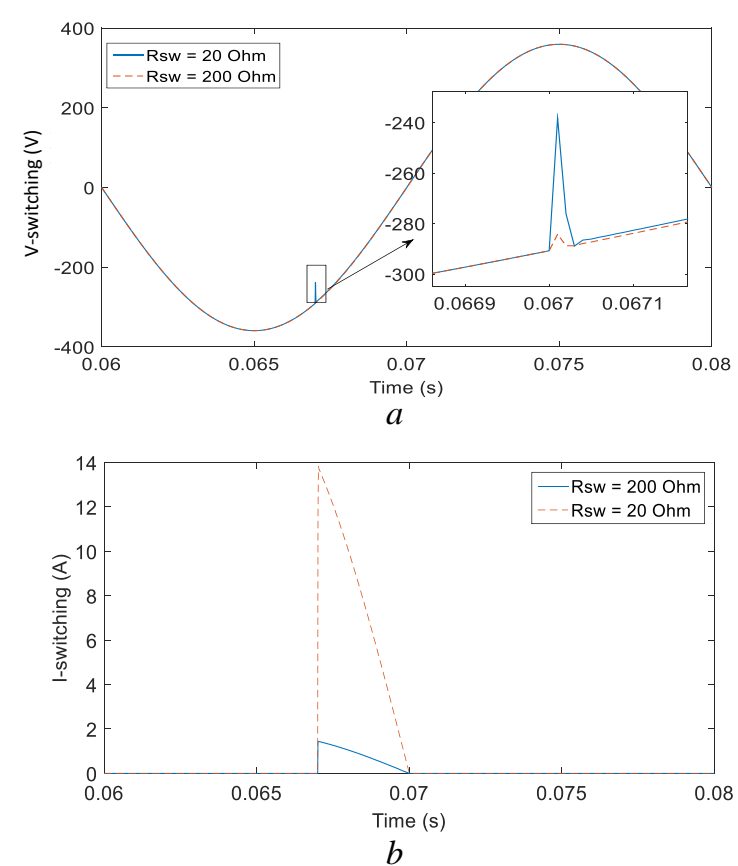

Fig. $4 \mathrm{~V}_{\text {switch }}$ and $\mathrm{I}_{\text {switch }}$ with $\mathrm{R}_{\mathrm{f}}=0.1 \Omega$ and different switching resistances (a) Switching voltage, (b) Switching current

Different simulation scenarios were performed to verify both the original simple approach and then the compensated approach with high system loads. Ground faults in three locations were applied to phase A of the simulated system of Fig. 3. The switching resistance used in this scenario was $200 \Omega$ (to limit the injection current), and the load was $10 \mathrm{~kW}$. 
Table 1 The parameter values for the simulated system

\begin{tabular}{lc}
\hline Parameter & Value \\
\hline Voltage & $440 \mathrm{~V}, 50 \mathrm{~Hz}$ \\
$\mathrm{Z}_{\mathrm{s}}$ & $0.0011+\mathrm{j} 0.0096 \Omega$ \\
$\mathrm{R}_{\text {line }} / \mathrm{m}$ & $30 \mu \Omega / \mathrm{m}$ \\
$\mathrm{L}_{\text {line }} / \mathrm{m}$ & $0.24 \mu \mathrm{H} / \mathrm{m}$ \\
Load & $10-100 \mathrm{~kW}$ \\
Zearth & $100 \mathrm{k} \Omega$ \\
\hline
\end{tabular}
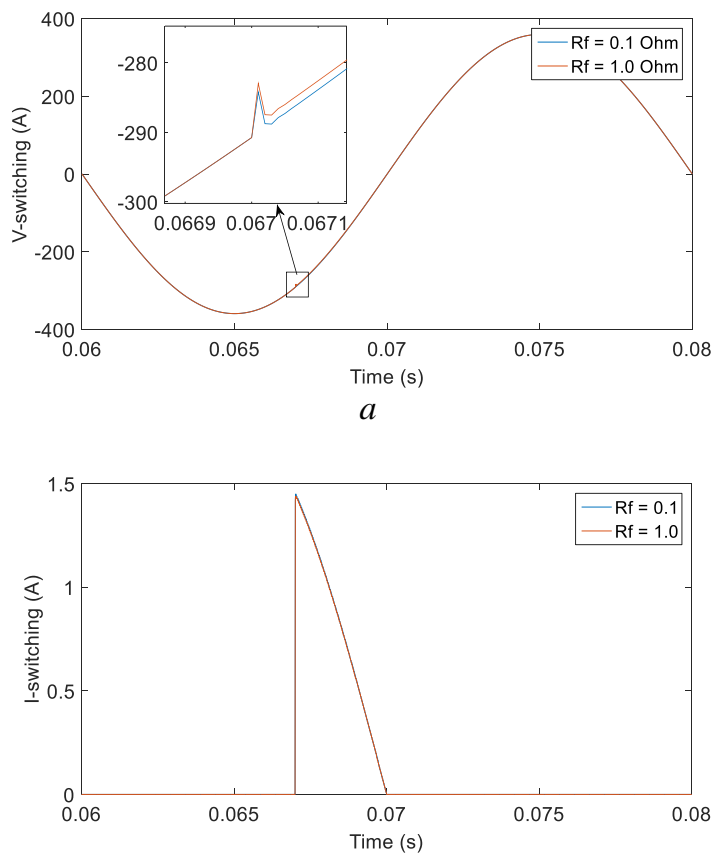

$b$

Fig. $5 V_{\text {switch }}$ and $I_{\text {switch }}$ with $R_{\text {switch }}=200 \Omega$, for different fault resistances (a) Switching voltage, (b) Switching current

Fig. 6 presents the estimated reactance for three locations, $0.0 \mathrm{~m}$ (S-end), $30 \mathrm{~m}$ and $50 \mathrm{~m}$ compared to the actual reactance of the line to these fault locations. A third line is included for each location which shows a curve fit of the estimated reactance using a first order least square curve fitting technique. It is clear that the technique offers a high accuracy for which the highest error was $4.6 \%$, occurring when the fault is located at the end of the line. The accuracy could be increased if a lower switching resistance is used which will create higher magnitude voltage and current transients and therefore a better SNR. For example, the error reduced to $1.45 \%$ when $R_{\text {switch }}$ is reduced to $20 \Omega$. Note that the estimated distance is the average of all the possible distances over the frequency range used (between $250 \mathrm{~Hz}$ and $3 \mathrm{kHz}$ ) [15]. The error is calculated based on the curve fitted line using (9):

$$
\text { error }=\frac{\text { Estimated distance-Actual distance }}{\text { Line length }} * 100(\%)
$$

The second scenario was to study the algorithm robustness against load change. Hence with the same system conditions, the load is increased to $100 \mathrm{~kW}$. The reactance estimation presented in Fig. 7(a) shows that the accuracy reduces

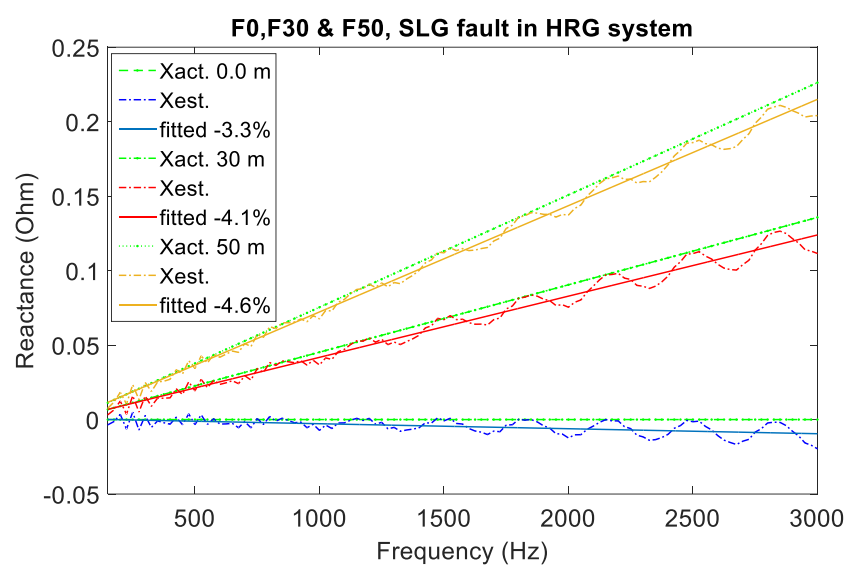

Fig. 6 Estimated reactance with $R_{\text {switch }}=200 \Omega, R_{f}=0.1 \Omega$

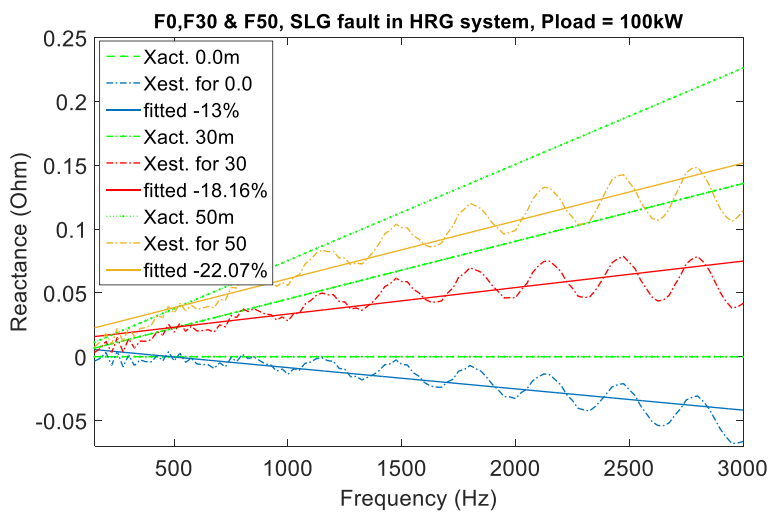

a

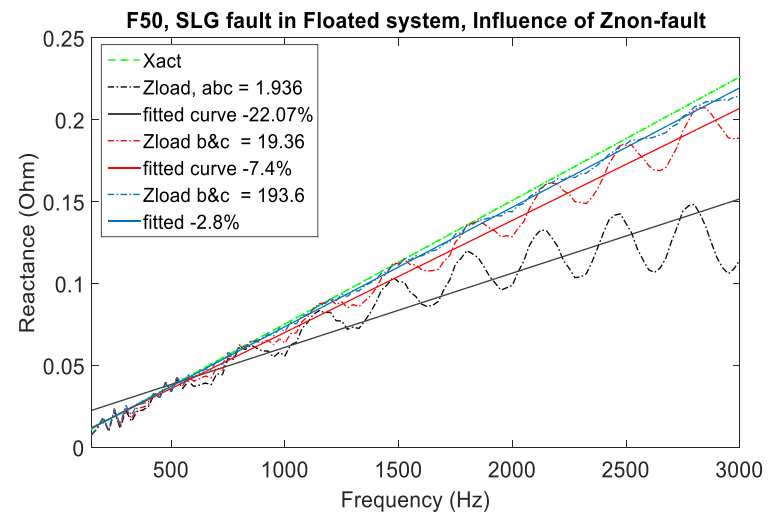

b

Fig. 7 The estimated reactance using (3), (a) balanced three phase load of $100 \mathrm{~kW}$ (b) The healthy phases load is changed to $10 \mathrm{~kW}$ and then $1 \mathrm{~kW}$.

significantly with a maximum error of $22 \%$ for the same fault conditions initiated in the previously described scenarios. To further examine the reason behind this divergence in accuracy, the switched current pulse was examined and it had been found that the majority of the transient pulse flowed through the healthy phases. This is because, when the load power increases, the load resistance decreases and hence, the equivalent impedance of the non-faulted phases reduced and becomes comparable to the faulted phase total impedance. A simulation was carried out to verify this theory. The healthy phase powers were reduced to $10 \mathrm{~kW}$ and $1 \mathrm{~kW}$ while the faulty phase power was kept the same at $100 \mathrm{~kW}$. Fig. 7(b) shows the 
influence of the load resistance for a fault at $50 \mathrm{~m}$. This significant outcome shows that the error was due to the equivalent impedance of the non-faulty phases and loads and the neglected part of the transient flowing through them when (3) is used to calculate fault location.

A third set of simulations was performed to demonstrate the compensation algorithm developed in Section 2.2. The same fault and system conditions were utilised to verify the proposed technique. The results obtained from applying the iterative method of equations (7) and (8) are shown in Fig. 8. These three estimated reactances indicate that the proposed algorithm can reliably compensate the influence of the equivalent impedance of the non-fault phases. The error is potentially reduced for a fault at the end of line from $22 \%$ to almost 5\%. A similar enhancement is achieved for the other two faults. The algorithm mostly needs three iterations to converge to the pre-set tolerance which in this case is $0.5 \mathrm{~m}$. Note the calculated error is presented on the figure legend for the curve fitted using a first-order least square method while the fluctuating line is the actual estimated reactance.

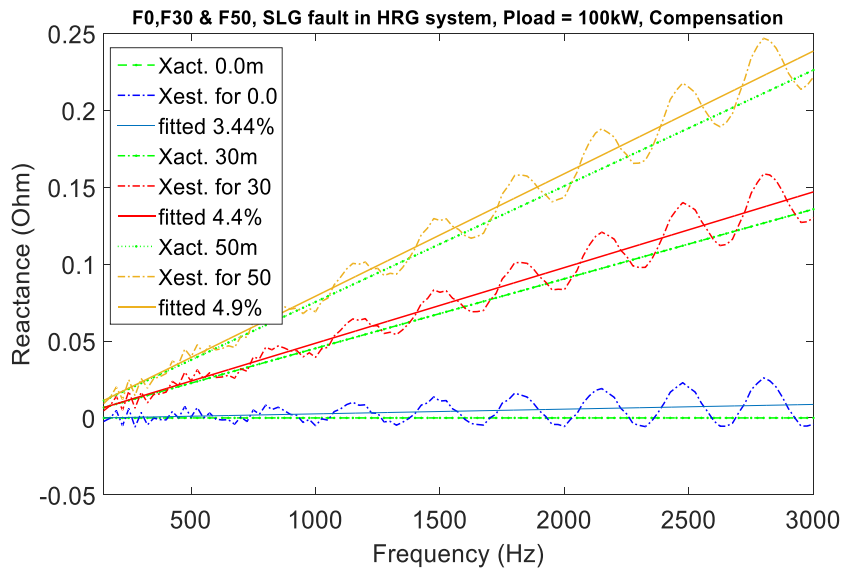

Fig. 8 The estimated reactance using the compensation algorithm of equations (7) and (8) - errors indicated for a curve-fitted reactance estimate

\section{Conclusion}

The location of a FGF in a HRG power system such as a marine power system is very important to prevent damage which may occur in the event of a second ground fault. In this work, the voltage measured on the grounding resistance is used to detect the FGF. Then after $10 \mathrm{~ms}$, a small resistance of $200 \Omega$ is connected in parallel to the HRG for $30 \mu \mathrm{s}$ in order to create a voltage and current transient which can be used to locate the FGF. Although these voltage and current transients were kept small in order to protect the system, the algorithm showed a reliable accuracy with a maximum error of $5 \%$. Moreover, the fault resistance has a very small influence on the proposed algorithm. However, the method is influenced by the equivalent impedance of the healthy phases which is dominated by the load impedance. The error increased significantly to $22 \%$ when the load increased from $10 \mathrm{~kW}$ to $100 \mathrm{~kW}$. A new solution was derived and used to compensate the effect of the non-fault phases. The simulation results showed that the algorithm is reliable and capable of compensating for the influence of the transient current passing through the non-fault phases.

\section{References}

[1] Bridger, B.: 'High-resistance Grounding' IEEE Transactions on Industry Applications, 1983, 19, (1), pp.1521.

[2] Nelson, J. P.: 'High-Resistance Grounding of LowVoltage Systems: A Standard for the Petroleum and Chemical Industry' IEEE Transactions on Industry Applications, 1999, 35, (4), pp 941-948.

[3] Das, J. C., Osman, R. H.: 'Grounding of AC and DC LowVoltage and Medium-Voltage Drive Systems' IEEE Transactions on Industry Applications, 1998, 34, (1), pp $205-$ 216.

[4] Baldwin, T., Renovich, F.: 'Analysis of Fault Locating Signals for High-Impedance Grounded Systems' IEEE Transactions on Industry Applications, 2002, 38, (3), pp.810817.

[5] Pan, Y., Silveira, P. M., Steurer, M., et al.: 'A Fault Location Approach for High-Impedance Grounded DC Shipboard Power Distribution Systems'IEEE Power and Energy Society General Meeting - Conversion and Delivery of Electrical Energy in the 21st Century, Pittsburgh, PA, USA, 2008.

[6] Pan, Y., Steurer, M., Baldwin, T.: 'Ground Fault Location Testing of a Noise Pattern based Approach on an Ungrounded DC System' IEEE Transactions on Industry Applications, 2011, 47, (2), pp. 996 - 1002.

[7] Pan, Y., Silveira, P. M., Steurer, M., et al.: 'Method for locating phase to ground faults in dc distribution systems'.

U.S. Patent 8067 942, Nov. 29, 2011.

[8] Ford, B., Leonard, I., Bosworth, M., \& Steurer, M.:'Grounding and Fault Location in Power Electronic based MVDC Shipboard Power and Energy Systems' In IEEE Electric Ship Technologies Symposium (ESTS), VA, USA, 2017, pp. 359-366.

[9] Wei, L., Liu, Z., Kerkman, R. J., Skibinski, G. L.: 'Identifying Ground-Fault Location Using Adjustable Speed Drive in High-Resistance Grounded Systems' IEEE Industry Applications Magazine, 2013, 19, (2), pp. 47 - 55.

[10] Hu, H., Wei, L., McGuire, J., Liu, Z.: 'Ground Fault Location Self-Diagnosis in High Resistance Grounding Drive Systems' IEEE Energy Conversion Congress and Exposition (ECCE), Pittsburgh, PA, USA, 2014, pp. 3179- 3185.

[11] Dubey, A., Sun, H., Nikovski, D., et al.: 'Locating of Multi-phase Faults of Ungrounded Distribution System' International Conference on Power System Technology (POWERCON 2014), Chengdu, China, 2014, pp. 1657-1664. [12] Jia, K. Thomas, D.W.P., Sumner, M.: 'Impedance-based earth fault location for a non-directly grounded distribution systems' IET Gener. Transm. Distrib., 2012, 6, (12), pp. 1272 1280.

[13] Cuzner, R. M., Sielicki, ., Archibald, A. E., McFarlin, D.A.: 'Management of Ground Faults in an Ungrounded Multi-Terminal Zonal DC Distribution System with 
Auctioneered Loads' IEEE Electric Ship Technologies Symposium (ESTS), Alexandria, VA, USA, 2011, pp. 300305.

[14] Patynowski, D., Cardenas, J., Menendez, D., et al.: 'Fault Locator Approach for High-Impedance Grounded or Ungrounded Distribution Systems using Synchrophasors' IEEE 68th Annual Conference for Protective Relay Engineers, College Station, TX, USA, 2015, pp.302-310.

[15] Jahanger, H. K., Sumner, M., Thomas, D.W.P.:' Combining Fault Location Estimates for a Multi-Tapped Distribution Line' IEEE PES Innovative Smart Grid Technologies Conference Europe (ISGT-Europe), Turin, Italy, 2017, pp. 1-6. 